\title{
Ipsilateral and contralateral masking of duration
}

\author{
HOWARD J. KALLMAN, JASON W. BECKSTEAD, and PATRICIA A. CAMERON \\ State University of New York, Albany, New York
}

\begin{abstract}
Judgments of the durations of test tones that were preceded or followed by a masking tone were elicited; the test tones and masks were presented either to the same ear or to different ears of the listeners. On both forward-and backward-masking trials, percentages correct increased with increases in the silent interstimulus interval (ISI) between the test tone and mask, an effect that did not depend on whether the mask was presented ipsilaterally or contralaterally. In addition, with increases in the ISI and with increases in the mask duration, there was an increasing tendency for listeners to respond "long" to the test tone, irrespective of the actual duration of the test tone. These biases were generally greater on trials in which the mask was presented ipsilaterally. It is suggested that, when stimuli occur in close temporal proximity, the decreased percentages correct at short ISIs may reflect misassignment of pulses generated by an internal clock. In contrast, the biases that result from manipulations of ISI and mask duration may be due to later operations, such as those that occur at the level of very-short-term memory.
\end{abstract}

The perception of time has received much recent attention. For example, two recent conferences have focused on this topic (see Gibbon \& Allan, 1984; Michon \& Jackson, 1985). Within the time-perception literature, an important research area is the perception of the temporal extent of brief stimuli. Many researchers have worked on this issue, and there are now a number of theories of how temporal extent is discriminated (for reviews, see Allan, 1979; Allan \& Kristofferson, 1974; Macar, 1985; see also Eisler, 1981; Kristofferson, 1977, 1980; Massaro, 1984; Massaro \& Idson, 1976; Thomas \& Cantor, 1975, 1978).

Experiments on duration masking have played an important role in the development of two often-cited theories of duration perception, one of which is based on work in the visual modality (Cantor \& Thomas, 1976) and the other on work in the auditory modality (Idson \& Massaro, 1977, 1980; Massaro \& Idson, 1976, 1978). Both theories maintain that the perception of duration is a function not only of the stimulus duration itself, but also of the amount of processing applied to the stimulus. And both theories were developed under the assumption that a backward mask, whether auditory or visual, could be used to control processing time. In a typical durationmasking experiment, the task is to judge the duration of a target stimulus that is either followed (backward masking) or preceded (forward masking) by another stimulus. With backward masking the assumption has been that the effect of the mask is due, at least in part, to termination of processing of the target stimulus.

Massaro and Idson (1976, 1978; Idson \& Massaro, 1977,1980 ) found that the perceived duration of a test tone tended to increase as the interstimulus interval (ISI)

Requests for reprints should be addressed to Howard J. Kallman, Psychology Department, State University of New York at Albany, 1400 Washington Avenue, Albany, NY 12222. between it and a backward mask increased up to about $250 \mathrm{msec}$. To explain this, they proposed that these increases in subjective duration reflected the additional time spent on processing the test tone given a long ISI. The notion of interruption of processing was also invoked by Massaro and Idson to explain their finding that the overall accuracy of test-tone categorizations increased with increases in ISIs, a result also reported by Allan and Rousseau (1977); Massaro and Idson suggested that as more stimulus information is processed, the short and long test tones become more discriminable. Although the bias to respond "long"' and the increase in percentages correct both result from increasing the ISI, Massaro and Idson (1976) demonstrated that these two effects were separable experimentally, and thus were not dependent on one another. ${ }^{1}$

Recent comparisons of forward and backward masking of duration challenge Massaro and Idson's view that the effect of the mask on duration perception is due to interruption of target or test-tone processing. Kallman and his associates (Kallman, Hirtle, \& Davidson, 1986; Kallman \& Morris, 1984) found that forward masks had virtually the same effects on judgments of the duration of test tones as backward masks, a result that is problematic to the interruption-of-processing view. This symmetry between forward and backward duration masking contrasts with the results of experiments on other auditory attributes, for example pitch and lateralization, in which backward-recognition-masking effects were found to be much greater than corresponding forward-masking effects (Massaro, 1973; Massaro, Cohen, \& Idson, 1976; Sparks, 1976).

Kallman et al. (1986) suggested that rather than reflecting interruption of processing, duration masking effects might be due largely to difficulty in separating out durational information about events that occur close together in time, irrespective of their temporal order. Therefore, 
the effects of a forward and of a backward mask are similar. Although this view represents a sort of integration theory, it differs from other versions of integration theory in that what is presumed to be integrated across stimuli is restricted to information about stimulus duration.

At this point, it is necessary to learn more about the variables that affect forward and backward duration masking to motivate, as well as place constraints on, additional theory development. Most of the research conducted to date was designed within the framework of an interruption theory of duration masking, and the experiments have tended to test assumptions of that sort of theory. Given the failure of interruption theory to explain recent duration-masking results, and the need to elaborate alternative theories, further specification of the conditions under which duration-masking effects obtain is needed.

The present duration-masking experiment was patterned after that by Kallman et al. (1986). However, the presentation ears of test tones and masks were varied, something that had not been done in previous duration-masking experiments. We were interested in whether the masking effects would differ as a function of whether the test tone and mask were presented to the same or to different ears. In pitch-masking studies, this sort of manipulation has proven helpful in establishing whether masking effects are due to processes that occur in the peripheral auditory system or, instead, centrally, that is, after input from the two ears is combined in the nervous system. If masking effects occur irrespective of whether the mask is presented ipsilaterally or contralaterally, the masking effects are necessarily due to central processing.

In addition to the issue of central versus peripheral processes, manipulation of the mask presentation-ear variable in pitch-masking studies has proven to be diagnostic with respect to the role of attentional and related processes on masking (Hawkins \& Presson, 1977, 1986; Kallman $\&$ Morris, 1984). Therefore, in the present experiment, both forward and backward masking of auditory duration were evaluated under contralateral and ipsilateral maskpresentation conditions.

\section{METHOD}

\section{Subjects}

Sixteen students at the State University of New York at Albany participated in four sessions, each approximately $1 \mathrm{~h}$ long. Fourteen of the listeners earned credit in an introductory psychology course, and 2 were paid $\$ 4$ per hour for their participation. Eight were assigned to the backward-masking condition and 8 to the forward-masking condition. All listeners reported no history of hearing disorder. Testing was done individually in an IAC soundinsulated chamber.

\section{Apparatus and Stimuli}

The stimuli were generated by Coulbourn Instruments auditory modules under the control of a Commodore- 64 computer, which also collected responses and presented visual feedback. All tones were $700-\mathrm{Hz}$ sinusoids with rise/fall times of $2 \mathrm{msec}$ and were presented through TDH-50 matched headphones. The sound levels of the tones were adjusted using a Brüel \& Kjaer Model 2203 soundlevel meter and Type 4152 artificial ear.

\section{Design and Procedure}

The listener's task was to indicate, by pressing one of two buttons on a numeric keypad, whether a test tone, which had a full amplitude duration of either 55 or $85 \mathrm{msec}$, was relatively short or long. The test tone was presented to the listener's left or right ear (according to a random schedule), and was either preceded (forward masking) or followed (backward masking), after a silent ISI of $5,25,45,105,165,205,255$, or $500 \mathrm{msec}$, by a masking tone. The mask had a full-amplitude duration of 40,70 or $100 \mathrm{msec}$, and was presented to the right or left ear (also according to a random schedule). To minimize the utility of loudness cues on the durationdiscrimination task, the intensity of each tone was randomly varied from among the integer values 75 through $81 \mathrm{~dB}$ (SPL).

The response interval, which began at the offset of the target tone, lasted $1.5 \mathrm{sec}$. Feedback, indicating whether the tone was short or long, was presented visually over the computer monitor for $500 \mathrm{msec}$. There was a 1.5 -sec intertrial interval.

In each of the four sessions, a block of 80 single-tone trials preceded the masking trials. The procedure and task were identical to the masking-tone trials except for the absence of the mask. The 80 single-tone trials were followed by two blocks of 384 masking trials, separated by a short rest period. There were 192 experimental conditions (test-tone durations $\times$ test-tone ear $\times$ ISI $\times$ mask duration $\times$ mask ear $[2 \times 2 \times 8 \times 3 \times 2])$; within each trial block, each condition occurred twice and the order of conditions presented was random.

\section{RESULTS}

An analysis of variance was conducted on the percentages correct. Test-tone duration, mask duration, ISI, and contralateral versus ipsilateral mask presentation (referred to hereafter as mask ear) served as within-subjects variables. Mask position (forward vs. backward mask) served as a between-subjects variable. The degrees of freedom reported for each test reflect the Huynh and Feldt (1976) correction for violations of sphericity.

The percentages correct as a function of ISIs for contralateral- and ipsilateral-mask trials are presented in Figure 1; forward-masking data are presented in the left panel and backward-masking data in the right.

Percentages correct increased with increases in ISI $[F(2.06,26.77)=47.70, p<.001, M S e=237.99]$; the lack of an ISI $\times$ mask position interaction $(F<1)$ indicates that the effect of ISI did not differ for forward-and backward-masking trials. Although mean performance was approximately $2 \%$ higher on contralateral than on ipsilateral forward-mask trials, the effect of mask ear was not significant $[F(1,13)=3.63, p>.05, M S e=243.46]$ and mask ear did not interact with mask position $(F<1)$. Neither the interaction between mask ear and ISI nor that between mask ear, ISI, and mask position were significant $(F<1, M S \mathrm{e}=107.02$, in both cases $){ }^{2}$ In sum, neither mask position nor mask ear had a significant effect on the discriminability of the short and long test tones, as measured by the average percentages correct.

Percentages correct as a function of ISI are presented in the panels of Figure 2, with separate panels for forward- and backward-masking trials; the curves are conditionalized on the various combinations of test-tone duration and mask ear. In every case, percentages correct at short ISIs were higher with short than with long test 


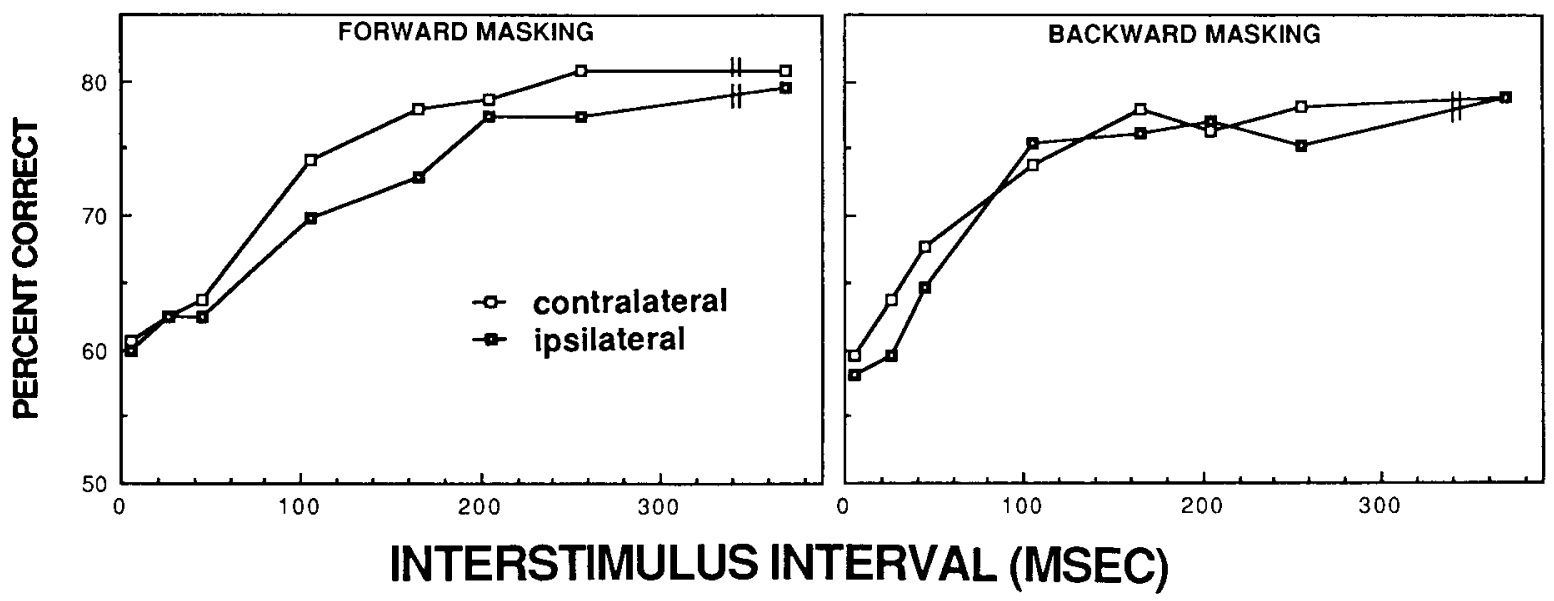

Figure 1. Percentages correct as a function of interstimulus interval for contralateral and ipsilateral masks. The two panels present data from forward-mask and backward-mask trials, respectively. In this and the following figures, the rightmost data points represent performance at an ISI of 500 msec.

tones; the opposite was true at the longer ISIs. A significant interaction between test-tone duration and ISI $[F(1.49,19.42)=11.30, p<.01, M S e=1,064.91]$, which did not interact with mask position $[F(1.49,19.42)$ $=2.05, p>.10, M S \mathrm{e}=1,064.91]$, confirms the reliability of this effect. As has been argued previously (Kallman et al., 1986; Massaro \& Idson, 1976), the increases in percentages correct with increases in ISIs that occur on long-test-tone trials reflect both (1) the tendency at long ISIs to hear the test tone as relatively long (which results in an increased number of responses of "long") and (2) increases in the discriminability of the short and long test tones. In contrast, on short-test-tone trials, these two factors-increases in discriminability and a bias to perceive the test tone as long-tend to cancel out one another, with the result that performance does not change much with increases in ISIs.
The size of the interaction between test-tone duration and ISIs was greater with ipsilateral than with contralateral masks $[F(3.40,44.25)=4.50, p<.01, M S \mathrm{e}=326.15]$. Although the size of this interaction did not differ significantly between forward- and backward-masking conditions $[F(3.40,44.25)=1.85, p>.10, M S e=326.15]$, visual inspection suggests that the three-way interaction was due primarily to the data from backward-mask trials. In sum, then, the biases due to the manipulations of ISI were present in all conditions (i.e., for all levels of mask position and mask ear). However, the bias appeared to be greatest with ipsilateral backward masks.

Percentages correct as a function of mask duration and ISI appear in the panels of Figures 3 and 4, which present data from short- and long-test-tone trials, respectively. The panels are conditionalized on the mask-position and mask-ear variables. Although neither the main effect of

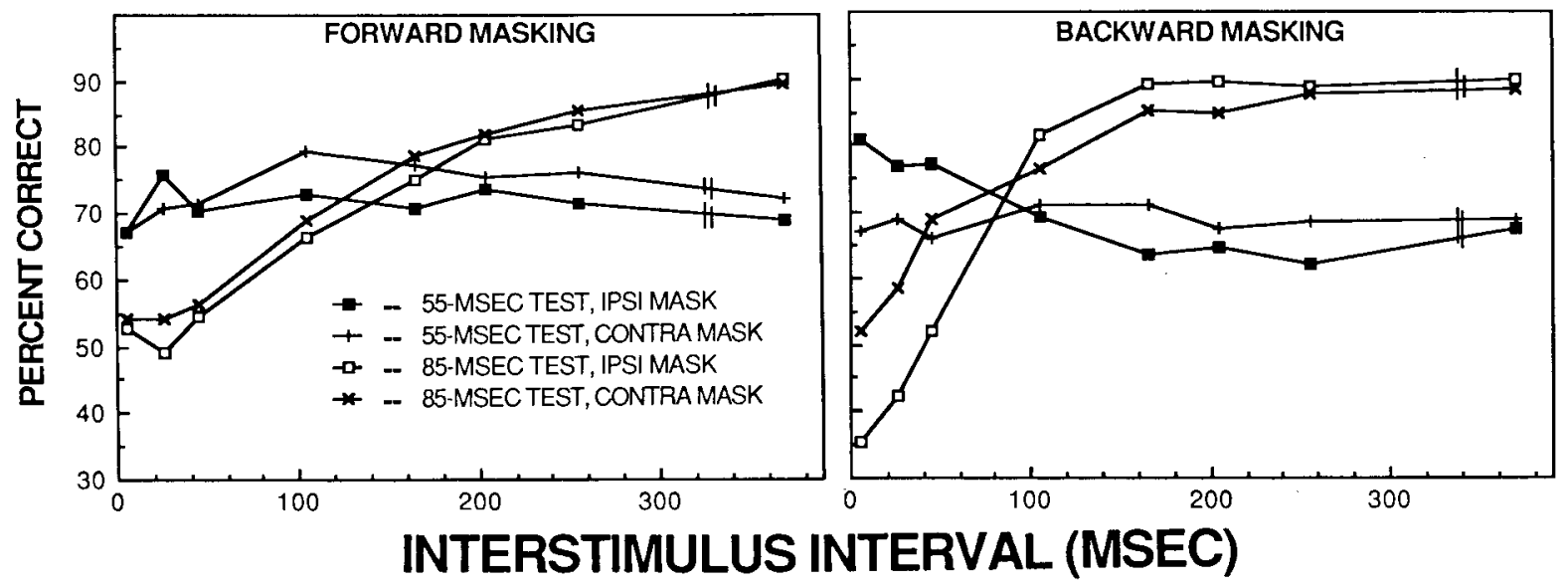

Figure 2. Percentages correct as a function of interstimulus interval; the curves are conditionalized on the duration of the test tone and whether the mask was presented ipsilaterally or contralaterally. 

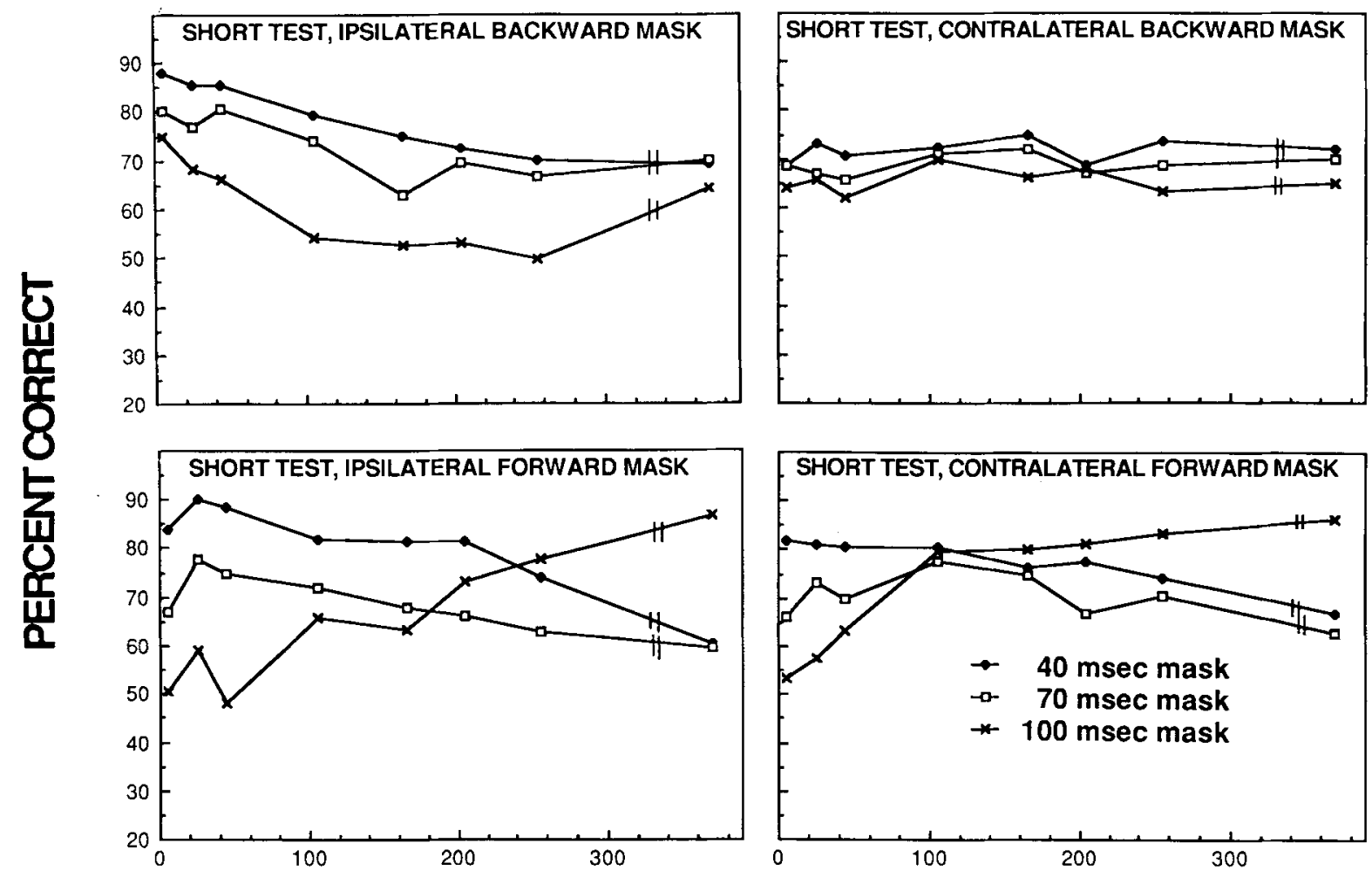

INTERSTIMULUS INTERVAL (MSEC)

Figure 3. Percentages correct for the short test tones as a function of interstimulus interval. The separate curves plot performance for each mask duration, and each panel represents performance for a particular combination of mask ear and mask position.

mask duration $(F<1, M S e=288.88)$ nor its interaction with mask position $[F(3.40,44.25)=2.08, p>.10$, $M S e=326.15]$ was significant, there was a significant interaction between test-tone duration and mask duration $[F(1.65,21.50)=18.20, p<.001, M S e=573.29]$, such that a correct response of "long" to a long test tone was more likely given a long mask, with the opposite being true for a short test tone. This effect, which was also found by Massaro and Idson (1976) and Kallman et al. (1986), can be appreciated by comparing the orderings of the three curves in the long-test-tone panels with those in the shorttest-tone panels; the orderings are generally reversed when the long- and short-test-tone conditions are compared. This is particularly so for performance with the ipsilateral masks.

The interaction between test-tone duration and mask duration did not differ significantly for forward- and backward-masking trials $[F(1.65,21.50)=1.15$, $p>10, M S \mathrm{e}=326.15]$. However, the interaction between test-tone duration and mask duration was greater on ipsilateral than on contralateral trials $[F(1.57,20.44)$ $=12.51, p<.001, M S e=200.12]$, an effect that did not depend on mask position $(F<1)$.

There were additional interactions that involved mask duration. The interactions between mask duration and ISI and between mask duration, ISI, and test-tone duration were significant $[F(14,182)=2.42, p<.05, M S e=$ 69.58 , and $F(12.20,158.58)=13.20, p<.001, M S e=$ 95.04, respectively]. However, both of these interactions depended on mask position $[F(14,182)=3.59, p<.01$, $M S \mathrm{e}=69.58$, and $F(12.20,158.58)=7.39, p<.001$, $M S e=95.04$, respectively], such that they were present only on forward-masking trials. ${ }^{3}$ These higher order interactions appear to reflect two general trends in the data. First, for the various combinations of mask position and ISI, the biasing effect of the mask was greatest on shortISI forward-mask trials. Second, performance on shorttest-tone forward-mask trials followed different trends for the 100-msec mask and the 40- and 70-msec masks; although, with either 40 - or 70 -msec masks, percentages correct on the short-test-tone trials decreased slightly with increases in ISIs, with the 100 -msec mask, percentages correct increased with increases in ISI. A similar trend was also evident in the forward-masking data from an earlier experiment conducted in our laboratory, although when that experiment was reported it was not clear that the trend was reliable (Kallman et al., 1986, Figure 3).

There was a significant interaction between mask duration, ISI, test-tone duration, and mask ear $[F(9.25,120.23)=2.45, p<.05, M S e=87.19]$; the 

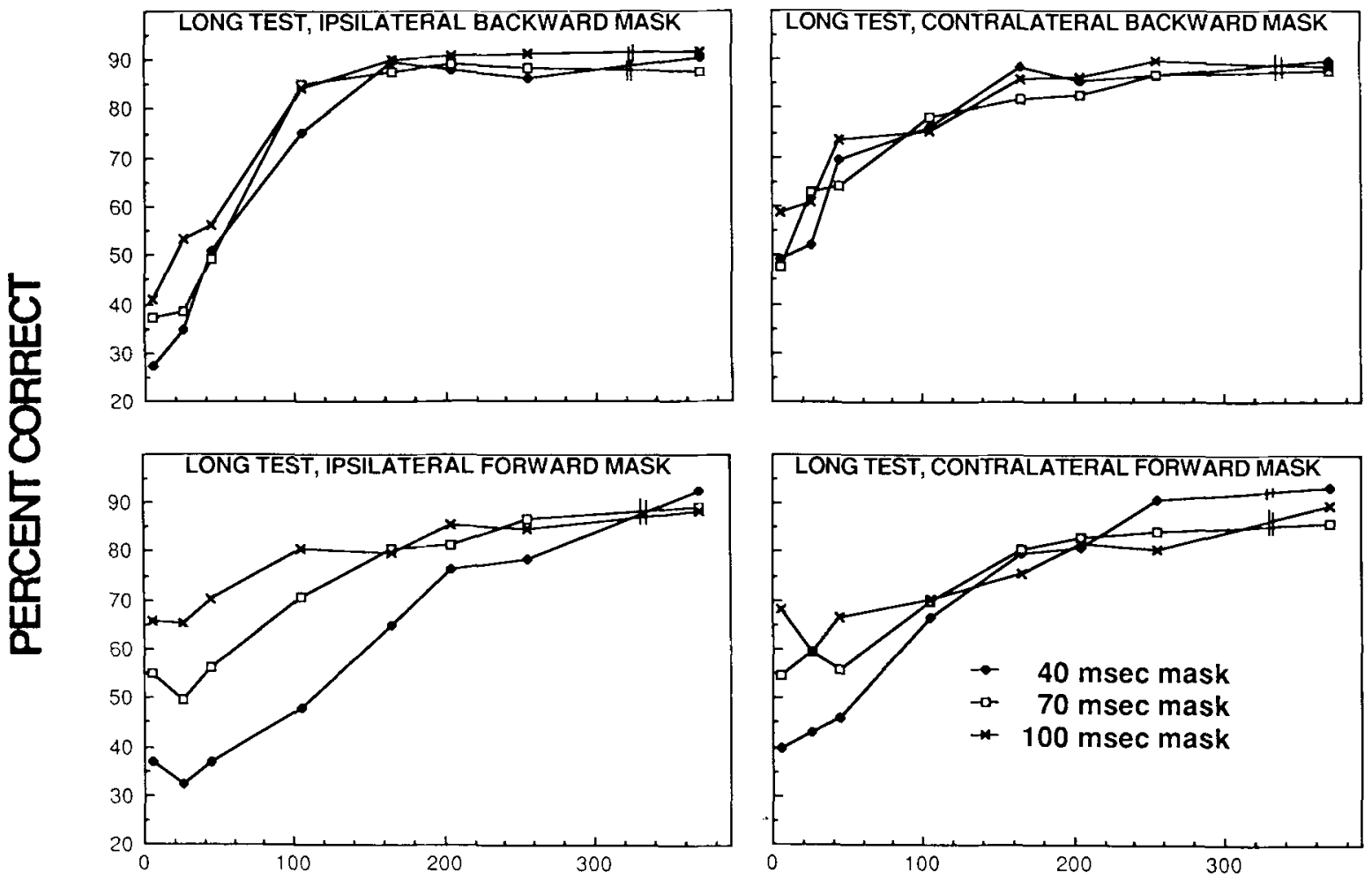

\section{INTERSTIMULUS INTERVAL (MSEC)}

Figure 4. Percentages correct for the long test tones as a function of interstimulus interval. In other respects, the data are organized as in Figure 3.

biasing effects of the mask durations were greatest with ipsilateral masks and short ISIs; this interaction did not differ significantly for forward and backward masking $(F<1)$.

In general, then, the biases brought about either by the ISI or the mask duration were present on both ipsilateraland contralateral-mask trials. However, for the most part, the biases were greater for the ipsilateral than the contralateral masks.

\section{DISCUSSION}

The present experiment was designed to evaluate the effect of presentation ear on the masking of auditory duration. The experiment also provided an opportunity to replicate, using procedures that were similar but not identical to those of earlier experiments, some of the previously reported duration-masking results and, in so doing, a test of their robustness.

Healthy increases in overall percentages correct with increases in ISIs were found under every combination of mask ear and mask position. This finding suggests that the discriminability effects obtained in duration-masking experiments are due to central, rather than peripheral, processes. Furthermore, although interruption of process- ing has been used to explain centrally based backwardrecognition masking of auditory attributes other than duration, the great amount of forward masking found in the present duration-masking experiment as well as in earlier experiments (Kallman et al., 1986; Kallman \& Morris, 1984) argues against an interruption-based explanation of duration masking. As has been suggested previously (Kallman et al., 1986), it seems likely that the discriminability effects arise from unique operations of a central timekeeper, possibly a misassignment of pulses that occur during presentations of the targets and masks.

With regard to the biases brought about by the manipulations of ISI and mask duration, two general points can be made. First, the biases were generally present under both contralateral and ipsilateral mask-presentation conditions. Therefore, the biases must be due largely, if not entirely, to central processes. But many of the biases were greater with the ipsilateral masks, suggesting that the central processes that were responsible for the biases took into account the presentation-ear variable.

The tendency to respond "short" given a short ISI and "long" given a long ISI was greatest when a backward mask was presented ipsilaterally. This difference between ipsilateral- and contralateral-mask conditions is of particular interest inasmuch as the mask-presentation ear was 
unknown to the listener prior to the onset of the mask. Accordingly, on the backward-mask trials, mask ear could not have affected the processing that took place during the ISI, because the mask had not yet been presented and its characteristics could not have been predicted. Therefore, an account of the bias in terms of the amount of processing that was carried out during the ISI would appear to be inadequate. Indeed, any explanation of the bias that attributes it primarily to processing that occurred during the ISI would seem to be ruled out by the present results, because the magnitude of the bias on backwardmask trials depended on the conditions of the mask, which were revealed only after the ISI had passed.

Perhaps the trickiest data to interpret are those pertaining to mask duration. Previous research (e.g., Kallman et al., 1986; Massaro \& Idson, 1976) demonstrated a mask-induced bias such that "short" responses were more likely given relatively short-duration masks and "long" responses were more likely given relatively long-duration masks. The biasing effect of the mask appeared, in the present experiment, to be greater on forward- than on backward-masking trials, particularly when the mask was presented ipsilaterally. It is possible that on backwardmask trials, listeners were able to divert attention from the mask prior to its offset. If this were the case, the perceived durations of the 40-, 70-, and 100-msec backward masks might not have differed to the degree that they would have if attention had been directed toward them throughout their presentation. In contrast, because the forward mask preceded the test tone, attention could not have been diverted without compromising attention to the test tone. As a result, the perceived durations, or at least the registration of temporal information, may have differed to a greater degree for the forward masks than for the backward masks, and this could have accentuated the mask-induced biases on forward-mask trials.

Additionally, on backward-mask trials, the effect of mask duration on the bias remained roughly constant through ISIs of approximately $250 \mathrm{msec}$, but this was not the case on forward-mask trials. In fact, on short-test-tone forward-mask trials, the bias reversed itself after an ISI of $100-200 \mathrm{msec}$, such that at the longer ISIs, listeners were more likely to respond "long" with a short mask than with a long one, a trend that can also be seen in the Kallman et al. (1986) data. Possibly the mask has its biasing effect either by (1) adding to processes that accumulate information about the duration of the test tone, thereby increasing the perceived duration of the test tone, or (2) serving as an anchor to which the test tone is compared. The forward mask might act as an anchor when its presentation lies outside the window in which temporal information is integrated by the temporal processor. Accordingly, at long ISIs, the forward mask might not directly affect temporal resolution of the test tone, but instead might serve as an anchor for the duration judgment. Given such a situation, a listener might respond "short" to a long test tone if the test tone was preceded by a longer mask, because the test tone would sound short relative to the longer mask. On the other hand, this sort of con- trast effect at the long ISIs might not occur on backwardmask trials, because much of the processing leading to a decision about the test tone would have been completed by the time the backward mask was presented. But at the short ISIs on backward-mask trials, temporal information about the mask could still become confused with temporal information about the test tone, so that the central timekeeper might attribute some of the mask's duration to the test tone. As a result, with relatively short ISIs, a mask-assimilation effect could occur, irrespective of whether a forward or backward mask is presented, but a mask-contrast effect could occur only on forwardmasking trials and at long ISIs.

Kallman et al. (1986) suggested that duration masking might reflect, at least in part, a tendency for the temporal processor to misassign temporal information from one stimulus event to another, with the result that maskinduced biases as well as decreases in test-tone discriminability characterize temporal judgments made when masks occur in close temporal proximity to test stimuli. But, in addition, there is ample evidence that other factors, such as the amount of processing applied to a stimulus, may contribute to perceived duration (Cantor \& Thomas, 1976; Thomas \& Brown, 1974; Thomas \& Cantor, 1975, 1978). If perception of duration does indeed stem from a number of more or less independent processes, each of which could decrease discriminability and/or add biases to judgments of perceived duration, the different processes might differentially contribute to particular duration masking effects. For example, the relatively low overall percentages correct at short ISIs could reflect the misassignment of pulses from an internal counter to stimulus events that occur close together in time and the resulting decrease in the discriminability of the test tones. The probability of misassignment of pulses may be independent of whether the two stimuli arrive via the same channel or not; indeed, given that it takes time to resolve the lateralization of a tone (Massaro et al., 1976), it is reasonable that ear of presentation would not affect whether pulses are misassigned. On the other hand, the effect of ISI on the bias to respond "short" or "long" might reflect processes that take place after the target and mask have both been presented, as the effect of backward-mask presentation ear on this bias suggests. Presently, we have no good explanation of why a long ISI should result in a higher probability of a long response on both forward- and backwardmask trials, a matter to be addressed by future research. Finally, the biases brought about by manipulations of the mask duration could well represent an assimilation of mask and test-tone information in very-short-term memory, as has been suggested earlier by Massaro and Idson (1978). In such a case, ear could represent a dimension of similarity in memory that determines the degree of assimilation of target and mask temporal information.

\section{REFERENCES}

Allan, L. G. (1979). The perception of time. Perception \& Psychophysics, 26, 340-354. 
Allan, L. G., \& Kristofferson, A. B. (1974). Psychophysical theories of duration discrimination. Perception \& Psychophysics, 16, 26-34.

Allan, L. G., \& Rousseau, R. (1977). Backward masking in judgments of duration. Perception \& Psychophysics, 21, 482-486.

Cantor, N. E., \& Thomas, E. A. C. (1976). Visual masking effects on duration, size, and form discrimination. Perception \& Psychophysics, 19, 321-327.

EISLER, H. (1981). Applicability of the parallel-clock model to duration discrimination. Perception \& Psychophysics, 29, 225-233.

Gibbon, J., \& Allan, L. (Eds.) (1984). Timing and time perception. Annals of the New York Academy of Sciences, 423.

Haw'Kins, H. L., \& Presson, J. C. (1977). Masking and preperceptual selectivity in auditory recognition. In S. Dornic (Ed.), Attention and performance $V I$ (pp. 195-211). Hillsdale, NJ: Erlbaum.

Hawkins, H. L., \& PrEsson, J. C. (1986). Auditory information processing. In K. R. Boff, L. Kaufman, \& J. P. Thomas (Eds.), Handbook of perception and human performance: Vol. 2. Cognitive processes and performance (pp. 26-1, 26-64). New York: Wiley.

HUYNH, H., \& FELDT, L. S. (1976). Estimation of the Box correction for degrees of freedom from sample data in the randomized block and splitplot designs. Journal of Educational Statistics, 1, 69-82.

IDSON, W. L., \& Massaro, D. W. (1977). Perceptual processing and experience of auditory duration. Sensory Processes, 1, 316-337.

IDSON, W. L., \& MASSARO, D. W. (1980). The role of perceived duration in the identification of vowels. Joumal of Phonetics, $8,407-425$.

Kallman, H. J., Hiktle, S. C., \& Davioson, D. (1986). Recognition masking of auditory duration. Perception \& Psychophysics, 40 , 45-52.

Kallman, H. J., \& Morris, M. D. (1984). Duration perception and auditory masking. Annals of the New York Academy of Sciences, 423, 608-609.

KRISTOFFERSON, A. B. (1977). A real-time criterion theory of duration discrimination. Perception \& Psychophysics, 21, 105-117.

Kristofferson, A. B. (1980). A quantal step function in duration discrimination. Perception \& Psychophysics, 27, 300-306.

MaCAR, F. (1985). Time psychophysics and related models. In J. A. Michon \& J. L. Jackson (Eds.), Time, mind, and behavior (pp. 112130). New York: Springer-Verlag.

MASSARo, D. W. (1973). A comparison of forward versus backward recognition masking. Joumal of Experimental Psychology, 100, 434-436.

Massaro, D. W. (1984). Time's role for information, processing, and normalization. Annals of the New York Academy of Sciences, 423, 372-384.

Massaro, D. W., Cohen, M. C., \& IDson, W. L. (1976). Recognition masking of auditory lateralization and pitch judgments. Journal of the Acoustical Society of America, 59, 434-441.

Massaro, D. W., \& IDSON, W. L. (1976). Temporal course of perceived auditory duration. Perception \& Psychophysics, 20, 331-352.

Massaro, D. W., \& IDSON, W. L. (1978). Target-mask similarity in backward recognition masking of perceived tone duration. Perception \& Psychophysics, 24, 225-236.

Michon, J. A., \& JACKSON, J. L. (Eds.) (1985). Time, mind, and behavior. New York: Springer-Verlag.

SPARKS, D. W. (1976). Temporal recognition masking-or interference? Journal of the Acoustical Society of America, 60, 1347-1353.

Thomas, E. A. C., \& Brown, I. (1974). Time perception and the filledduration illusion. Perception \& Psychophysics, 16, 449-458.

Thomas, E. A. C., \& Cantor, N. E. (1975). On the duality of simultaneous time and size perception. Perception \& Psychophysics, 18, 44-48.

Thomas, E. A. C., \& Cantor, N. E. (1978). Interdependence between the processing of temporal and non-temporal information. In J. Requin (Ed.), Attention and performance VII (pp. 43-62). Hillsdale, NJ: Erlbaum

\section{NOTES}

1. The response biases referred to in this article might be due to decision processes operating at the response stage. Alternatively, they might reflect biases in how the stimuli are perceived or retained in very-shortterm auditory memory. In addition to the finding of biases in experiments in which a binary response task was used, Idson and Massaro (1977) found similar biases when responses were made on a continuous rating scale of perceived duration. Given that the biases persisted with this change in response type, we favor the view that the biases reflect perceptual and/or very-short-term memory phenomena rather than decision processes at the response stage. This issue is not fully resolved by the available data, however.

2. A separate analysis of variance (ANOVA) was performed on the forward-mask data alone to provide a more powerful test of whether there were differences in percentages correct between ipsilateral- and contralateral-mask conditions. In this analysis, both the main effect of mask ear $[F(1,7)=4.85, p>.05, M S \mathrm{e}=184.69]$ and the interaction between mask ear and ISI $(F<1)$ were not significant.

3. To confirm that the interaction between mask duration and ISI as well as the interaction between mask duration, ISI, and target duration were present on forward-mask trials but not on backward-mask trials, separate ANOVAs on the forward-mask and backward-mask data were examined. The forward-masking analysis showed that both the two-way interaction $[F(4.26,29.81)=4.12, p<.01, M S e=76.89]$ and the three-way interaction $[F(3.21,22.47)=17.94, p<.001, M S \mathrm{e}=$ 117.31] were significant. On backward-mask trials, neither the two-way nor the three-way interaction was significant $[F(12.65,88.57)=1.74$, $p>.05, M S \mathrm{e}=67.96$, and $F(14,98)=1.21, p>.10, M S \mathrm{e}=79.90]$

(Manuscript received February 5, 1987; revision accepted for publication July 10, 1987.) 